\title{
Oral Cytotoxic Chemotherapy Safe Use and Safe Handle Among Cancer Patients in Radiation and Isotopes Center, Sudan
}

\author{
Alaa Abdelaziz Mohamed, MSc. ${ }^{\text {a }}$, Mohamed Awad Mousnad, PhD ${ }^{\text {*b }}$ \\ ${ }^{a}$ Clinical Pharmacy, University of Khartoum, Sudan \\ ${ }^{\mathrm{b}}$ Assistant Professor of Pharmacy Practice at International University of Africa (IUA), Sudan \\ *Corresponding author: Mohamed Mousnad, is a Consultant of Pharmacoeconomics \& Pharmacoepidemiology, Assistant \\ Professor, Faculty of Pharmacy, International University of Africa (IUA), Khartoum, Sudan; m_abdalaziz@yahoo.com
}

Received 16 May 2019;

Accepted 02 June 2019;

Published 16 June 2019

\begin{abstract}
Background: Increasing use of oral cytotoxic chemotherapy among cancer patient in Sudan, rising important concern about safety practicing and using of these drugs. This study aimed to assess and demonstrate patient awareness regarding their oral cytotoxic drugs regimen in Radiation and Isotopes Center at Khartoum (RICK), as it considered one of the essential hospital cancer treatments in Sudan. Method: Acrosssectional study was carried out at (RICK) hospital outpatient pharmacies. Data were collected by interviewing 100 patients and answering

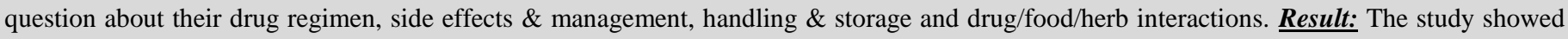
that $46 \%$ of participants have missing knowledge about their drug using and taking. Complaints reported by patients, when using their drugs, vary. Myelosuppression is the most common (40.2\%). In side effect management, the study showed that $26 \%$ of the patients are referred to the community pharmacy and $67 \%$ are using medication without a prescription. Regarding the awareness of using herbal treatments, $42 \%$ of patients are using or have used herbs with their drugs. $69 \%$ of patients using herbs said that they do not inform the caregiver. For the proper drug handling and storage in the home setting, $59.6 \%$ of patients said they did not receive instructions about handling and storage. The degree of patient knowledge about the proper handling and storage are variable. Finally, patient's knowledge about their drug regimen was significantly associated with drug education, an age of $13-45$ years and regularity of clinic visits $(p=0.018)$. Conclusion: The study concluded that awareness of patients is very low regarding the toxicity of these drugs and education of patients for oral cytotoxic chemotherapy vary substantially. The development of therapeutic educational programs is essential to ensure safe practice when using oral cytotoxic drugs.
\end{abstract}

Keywords: oral cytotoxic chemotherapy, antineoplastic, safe practicing, patient education, patient awareness, side effect management, handling and storage, food/herb/drug interaction.

\section{Introduction}

The administration of oral chemotherapy has been in use since as early as the 1940 s to treat chronic leukemia. ${ }^{[1]}$ After the approving of capecitabine to treat cancer at $199,{ }^{[2]}$ the development of oral chemotherapy agents has increased dramatically. ${ }^{[1,2]}$ With approving of new targeted molecular therapies, more than $25 \%$ antineoplastic agents currently being developed are oral drugs. ${ }^{[3,4]}$ These shifting oncologists to prescribe oral chemotherapy, as it has many social and economic advantages for patients including increased control and convenience for the patient, potential increase in the quality of life, sustained medication exposure, and potential reduction in travel costs and use of health care resources. ${ }^{[1]}$ While there are several advantages to prescribing oral chemotherapy, one must bear in mind that home-based chemotherapy may continue for some time without professional supervision. The intermittent nature of treatment regimens may be confusing to some patients and their families and noncompliance through misinterpretation carries the risk of serious harm. ${ }^{[5,6,7]}$

Oral cytotoxic chemotherapy has the same risk as parenteral chemotherapy in terms of toxicities and potential for harm from medication, due to the narrow therapeutics index of these drugs. ${ }^{[5,6,8]}$ Although there are few publications comparing chemotherapy errors that occur with oral versus intravenous administration, ${ }^{[9]}$ there are several concerns that arise, including patient adherence, management of adverse reactions, drugs interaction, storage and handling. Which creates challenges and increase responsibilities for healthcare professionals in patient education. ${ }^{[5,6,10]}$

Safe practicing of oral cytotoxic drugs involves the processes of prescribing, dispensing, patient education, administration, handling and storage of these drugs. ${ }^{[11]}$ This study concentrates on the safety 
practices for these drugs from the patient point of view and their home setting using. No recent study was done in Sudan that indicates safety of using these oral cytotoxic chemotherapies. The main objective of this study is to demonstrate safety practicing and using of oral cytotoxic chemotherapy by patient in RICK hospital in Sudan. To specifying this objective, study was assessing patient awareness regarding four categories: Drug regimen, Side effects \& management, Handling \& storage and Drug/food/herb interactions.

\section{Method}

This study is Qualitative, descriptive, cross-sectional study. It was carried out at the pharmacies of Radiation and Isotopes Center of Khartoum. Targeted all Patients on an oral cytotoxic chemotherapy regimen, excluding patients on oral hormonal chemotherapy, come to the clinics in February 2016.

\subsection{Data collection method}

Data were collected by using questionnaire, was administered by the researcher. It was designed based on those from the literature and the data from the drug information center (DIC) at RICK hospital. A pilot study was done to validate and adjust it.

\subsection{Sample selection}

It is a convenient sample because no basic data were found about the total numbers of targeted patients to calculate sample size. Therefore, patients on oral cytotoxic drugs who come to pharmacies to dispense their medicine during February were included in the study. The total number of patients was 100 .

\subsection{Study protocol}

It was collected by interviewing patients when they came to dispense their medicine on three days of the week (Sunday, Tuesday and Wednesday) according to the clinic for this targeted population.

\subsection{Data management and statistical analysis}

Data were tabulated using excel 2010, and analyzed using statistical package analysis of social science (SPSS v.20). Binary logistic regression analyses were carried out for the dependent variable ' knowledge of patient how to use his drugs'.

\subsection{Ethical considerations}

Approval from the hospital authorities and consultants running the pharmacies was obtained. Also, verbal informed consent was obtained from patients prior to data collection after giving them brief information about the study.

\section{Results}

\subsection{Demographic data of patients and visit regularity:}

Patients who participated in the study, 58\% were male and $42 \%$ were female. Their age varies $46 \%$ aged $46-65,22 \%>65$ years old, $17 \%$ 13-45 years, and $15 \%$ aged $<12$ years old. Educational level of participants were as follows: $41 \%$ were illiterate, $27 \%$ were primary, $23 \%$ were secondary and $9 \%$ were graduate or above.

Regarding the regularity of clinic visits, $59 \%$ of patients said they are come regularly, $27 \%$ not on regular visit and $12 \%$ of them were their first visit.

\subsection{Patients drugs education:}

For the verbal education, only $52 \%$ of patients said they received information, with the source of these being the pharmacist in
$67.3 \%$ of cases. While $98 \%$ said that they did not receive written instructions about their drugs. The study showed that $46 \%$ of participants have missing knowledge about their drug using and taking.

The association between knowledge of drug use and participant's education level showed that $61 \%$ of illiterate patients do not know how to take their drugs, $44.4 \%$ of those with a primary educational level, $34 \%$ with secondary level and $11.1 \%$ with graduate level.

\subsection{Side effects and managements:}

Complaints reported by patients, when using their drugs, vary. Myelosuppression is the most common (40.2\%), while nausea and other complaints such as diarrhea, infection sign and yellowish skin have comparable percentages.

In the management of these complaints (table 1) and (table 2), Unexpectedly, the study showed that $26 \%$ of the patients are referred to the community pharmacy and $67 \%$ are using medication without a prescription, like analgesics by $60.3 \%$ and antibiotics by $44.1 \%$. A correlation between the use of these medications and educational level showed that those with secondary education level are less likely to use these medications, while illiterate patients reported the highest percent by $78 \% .70 \%$ of patients using these medications do not inform their doctors or caregivers.

Table (1): Action taken by the participants when side effects developed

\begin{tabular}{|l|l|l|}
\hline Actions & Frequency & Percent \\
\hline Refer to community pharmacy & 26 & 26.0 \\
\hline Refer to your doctor at RICK hospital & 23 & 23.0 \\
\hline Stop taking medicine until next visit & 23 & 23.0 \\
\hline Refer to GP at other hospital & 17 & 17.0 \\
\hline First visit & 11 & 11.0 \\
\hline Total & 100 & 100.0 \\
\hline
\end{tabular}

Table (2): The use of medication without prescription among participants

\begin{tabular}{|c|c|c|}
\hline \multicolumn{3}{|c|}{ medication use } \\
\hline & Frequency & Percent \\
\hline Yes & 67 & 67.0 \\
\hline No & 33 & 33.0 \\
\hline Total & 100 & 100.0 \\
\hline \multicolumn{3}{|c|}{ Informing doctor about medication use } \\
\hline & Frequency & Percent \\
\hline Yes & 20 & 30.0 \\
\hline No & 47 & 70.0 \\
\hline Total & 67 & 100.0 \\
\hline
\end{tabular}

\subsection{The using of herbal among patients:}

Regarding the awareness of using herbal treatments, $42 \%$ of patients are using or have used herbs with their drugs. This practice was found most often in patients with a secondary educational level, illiterate and primary (47.8\%), (43.9\%) and (40.7\%) respectively. $69 \%$ of patients using herbs said that they do not inform the caregiver. Unexpected data found that $42.9 \%$ of caregiver advised to continue when informed about the traditional remedies.

\subsection{Drugs handling and storage:}

For the proper drug handling and storage in the home setting, $59.6 \%$ of patients said they did not receive instructions about 
handling and storage. On the other hand, study showed that most patients counseled about handling and storage said that they take information from pharmacists $(29.3 \%)$.

The degree of patient knowledge about the proper handling and storage showed that $54.8 \%$ know the storage conditions of their drugs. Other points of these are variable as shown in (figure 1) but the most important concern of it, is to avoid direct contact of these drugs with skin and avoiding tablet or capsule crushing. Result showed only $28.6 \%$ of patients know it and avoid contact by wearing gloves or using drug caps to swallow it; on the other hand, $4.8 \%$ of them know about avoiding crushing their medicine.

The logistic regression model assessed different patient variables (drug education, age 13-45 and regularity of clinic visit) with knowledge of drugs uses as seen in table (3). It showed significant interaction of the predictors (Chi-square $=47.896$, pvalue $=0.018$ ). This model showed predictive ability of the variation using these set of variables up to $47 \%$ of the variation in the knowledge of respondents.

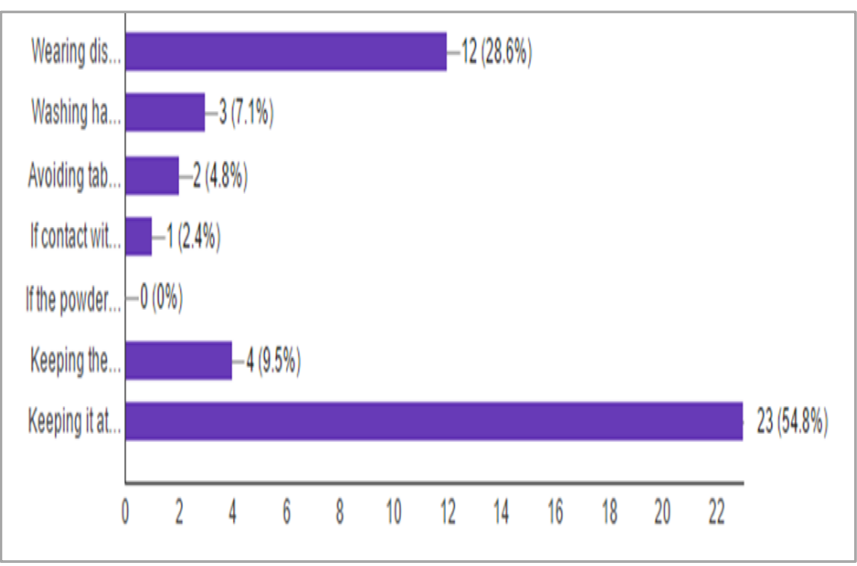

Figure 1: Degree of patient knowledge and proper handling in the home setting.

Table 3: Binary logistic regression model assessing predictors of knowledge of how to use medications among study participants

\begin{tabular}{|l|c|c|c|c|}
\hline & B & Wald & Sig. & Odd ratios \\
\hline Drug education & 1.960 & 10.868 & 0.001 & 7.100 \\
\hline Age of 13-45 & 1.777 & 3.946 & 0.047 & 5.910 \\
\hline $\begin{array}{l}\text { Regular visits to } \\
\text { caregivers }\end{array}$ & 1.275 & 3.899 & 0.048 & 3.580 \\
\hline Constant & -0.140 & 0.015 & 0.904 & 0.869 \\
\hline
\end{tabular}

\section{Discussion}

This study is one of fewer studies in Sudan that concerned to evaluate the safety in practicing and using of oral cytotoxic drugs among cancer patients.

According to the data collected from drug information center (DIC), at the RICK hospital, there are seven pharmacies dispensing chemotherapy, two of which were specified to dispense oral cytotoxic drugs. Pharmacies staff is shifted constantly through all pharmacies, so all of them dispensed oral cytotoxic drugs. The range of daily prescriptions seen by all staff including oral and IV was around 640-810.
The study found that illiterate patients represent the highest participants among the study group by $41 \% .59 \%$ of patients said they are come regularly to clinics as instructed by their doctors.

The association between knowledge of drug use and participant's education level showed that parallel relation as educational level decreased the knowledge decreased too. Therefore, the educational level has an effect on the knowledge of drug use.

The written instruction papers do not focused by caregivers, in which $98 \%$ said that they did not receive written instructions about their drugs. For the verbal education, $52 \%$ of them said they received information, with the source of these being the pharmacist in $67.3 \%$ of cases. When comparing these results with the literature ${ }^{[3,4,12,13]}$ it was found that most of them agreed about the important of initial visits, providing patients with educational materials and increasing their awareness about the need for regular monitoring arrangements as the recommendation stated by ASCO. In Simchowitz et al. (2010), in addition to the information provided by their clinicians, all participants reported seeking additional information from other sources, and pharmacists. Also, participants described positive interactions with their pharmacists and were helpful in educating them and improving the safety of oral chemotherapies. Participants believed that the initial prescribing encounter should have included more education. Bourmaud et al. (2014) stated that $54 \%$ of the centers provided educational materials for the patient at the first visit, while $12 \%$ of the clinicians who participated in this study reported regular workshops with their patients. In Conde-Estévez et al. (2013), $44.2 \%$ of hospitals classified as level II, and performed an initial visit with a pharmacist providing written patient educational materials and monitoring adherence. In the study published in the BMJ in 2007, they found that $95 \%$ of the patient education was by the pharmacist, while the physician shared responsibility for educating patient.

Regarding side effects, Complaints reported by patients vary. Myelosuppression is the most common (40.2\%). By looking to previously published studies, like Bourmaud et al. (2014), 39\% of the clinicians they said that they recalled at least one serious adverse event over one year and $80 \%$ of them said that they are worried about the risks of oral chemotherapy. In the management of these complaints, the study reported heterogeneity among participants, as shown in Simchowitz et al. (2010), the participants raised concerns regarding their lack of preparedness for side effects and their unfamiliarity with the possible techniques to mitigate drug toxicity.

The using of herbal treatments or dietary supplement reported to be common among participants by $42 \%$ with variation of their educational level.69\% of patients using herbs said that they do not inform the caregiver. Unexpected data found that $42.9 \%$ of caregiver advised to continue when informed about the traditional remedies. This may be due to a lack of proper counseling or may be because remedies were not expected to have clear interactions with drugs, such as the use of honey or common Sudanese remedies (gongolis, dom, and acacia). In Segal et al. (2014), drugfood interactions were noted for the 58 oral chemotherapeutics, so this study and ASCO (2013) stated that reviewing the current medications should be performed with the patient to identify potential medication interactions or interference with dietary requirements. 
The results of this study rising concern about the proper drug handling and storage in patients home setting. In which more than half of participants they do not received education about it. Beside that home setting handling represent low percentage of good handling among patients as shown in (figure 1). These results were nearly matched the outcome of Simchowitz et al. (2010), and Chan et al. (2009), which both demonstrated the need to improve patients' understanding of the requirements for storage, handling and the safe administration of oral cytotoxic chemotherapies.

The model in (table 3) showed odd ratios of prevalence pointed to possibility of having appropriate knowledge about drug use 7 times among who received education compared to those who were deficient in this. Interestingly the age group of 13-45 years showed 5 times greater ability to know their treatment compared to the other age groups. Regular visits to caregivers boosted the ability of candidates to confidently know the use of their drugs by 3.58 times compared to candidates who were not concerned about visit regularity.

\section{Conclusions}

The study indicated low consensus and awareness of patients toward the toxicity of oral cytotoxic chemotherapy and how to handle it in safe manner. This would increase demand toward patient education about their drugs regimen. Indeed, patient educational level as important factors during the first visit to get the goal of safe using and practicing of oral cytotoxic drugs.

\section{Acknowledgments}

This paper was part of a thesis at the University of Khartoum, Sudan submitted by Alaa Abdalaziz Mohamed and supervised by assist.Prof. Mohamed Awad Mousnad. The authors would like to thanks to thank faculty members, staff at faculty of Pharmacy, $U$ of $\mathrm{K}$ for their precious suggestions. Many thanks also goes to the RICK Hospital and Pharmacy staff for their cooperation.

\section{Declarations}

\section{Conflict of interest}

The Author(s) declare(s) that they have no conflicts of interest to disclose.

\section{References}

[1] Aisner J. Overview of the changing paradigm in cancer treatment: oral chemotherapy. American Journal of Health-System Pharmacy. 2007 May 1; 64(9).

[2] Karen L Milkiewicz, PhD, RPh Research Scientist II, Teva Pharmaceuticals, Oral Chemotherapy and the Pharmacist's Role: Providing Pharmaceutical Care for Cancer Patient, 2015, PharmCon, Inc. has been approved by the American Nurses Credentialing Center's Commission on Accreditation.

[3] Bourmaud A, Pacaut C, Melis A, Tinquaut F, Magné N, Merrouche Y, Chauvin F. Is oral chemotherapy prescription safe for patients? A cross-sectional survey. Annals of Oncology. 2014 Jan 9:mdt553.

[4] Conde-Estévez D, Salas E, Albanell J. Survey of oral chemotherapy safety and adherence practices of hospitals in Spain. International Journal of Clinical Pharmacy. 2013 Dec 1;35(6):1236-44.

[5] Grampians Regional Palliative Care Team, Clinical Guidelines for the Administration of Oral Chemotherapy Agents in the Community Setting For Health Care Professionals, 2013, Victoria.

[6] SHPA Committee of Specialty Practice in Cancer Services, Standards of Practice for the Provision of Oral Chemotherapy for the Treatment of Cancer. Journal of Pharmacy Practice and Research, 2007, Volume 37, No 2.

[7] Schulmeister L. Chemotherapy medication errors: Descriptions, severity, and contributing factors. Oncol Nurs Forum 26:1033-1042, 1999

[8] Goodin S, Griffith N, Chen B, Chuk K, Daouphars M, Doreau C, Patel RA, Schwartz R, Tamés MJ, Terkola R, Vadnais B. Safe handling of oral chemotherapeutic agents in clinical practice: recommendations from an international pharmacy panel. Journal of Oncology Practice. 2011 Jan 1;7(1):7-12.

[9] Blecher C, Barefoot J, Davis D, et al: A team approach toward promoting patient adherence to oral chemotherapy protocols. Oncol Nurs Forum 35:537, 2008 (abstr 2985).

[10] Carrington C. Safe use of oral cytotoxic medicines. Australian Prescriber. 2013 Feb; 36 (1).

[11] Royal Cornwall Hospitals NHS trust, The Safe Handling and Administration of Cytotoxic Products for the Treatment of Cancer. August 2015.

[12] Weingart SN, Flug J, Brouillard D, Morway L, Partridge A, Bartel S, Shulman LN, Connor M. Oral chemotherapy safety practices at US cancer centers: questionnaire survey. BMJ. 2007 Feb 22; 334(7590):407.

[13] Neuss MN, Polovich M, McNiff K, Esper P, Gilmore TR, LeFebvre KB, Schulmeister L, Jacobson JO. 2013 updated American Society of Clinical Oncology/Oncology Nursing Society chemotherapy administration safety standards including standards for the safe administration and management of oral chemotherapy. Journal of Oncology Practice. 2013 Mar $1 ; 9(2 \mathrm{~S}): 5 \mathrm{~s}-13 \mathrm{~s}$.

[14] Simchowitz B, Brouillard D, Maureen Connor RN MP, Weingart SN. Perceptions and experiences of patients receiving oral chemotherapy. Clinical Journal of Oncology Nursing. 2010 Aug 1;14(4):447.

[15] Segal EM, Flood MR, Mancini RS, Whiteman RT, Friedt GA, Kramer AR, Hofstetter MA. Oral chemotherapy food and drug interactions: a comprehensive review of the literature. Journal of Oncology Practice. 2014 Jul 1; 10(4):e255-68.

[16] Chan A, Leow YC, Sim MH. Patients' perspectives and safe handling of oral anticancer drugs at an Asian cancer center. Journal of Oncology Pharmacy Practice. 2009 Sep 1;15(3):161-5. 